OPEN ACCESS

Edited by:

Ahmed Negida,

Zagazig University, Egypt

Reviewed by:

Sayona John,

Rush University, United States Jorge Marcelo Mura, Instituto de Neurocirugía, Chile

*Correspondence: Xin Qu

quxin0212@163.com

Specialty section: This article was submitted to Neurosurgery,

a section of the journal

Frontiers in Surgery

Received: 02 June 2021 Accepted: 27 September 2021

Published: 26 October 2021

Citation:

Shang F, Zhao H, Cheng W, Qi M, Wang $N$ and Qu X (2021) Predictive

Value of the Serum Albumin Level on

Admission in Patients With

Spontaneous Subarachnoid

Hemorrhage. Front. Surg. 8:719226.

doi: 10.3389/fsurg.2021.719226

\section{Predictive Value of the Serum Albumin Level on Admission in Patients With Spontaneous Subarachnoid Hemorrhage}

\author{
Feng Shang, Hao Zhao, Weitao Cheng, Meng Qi, Ning Wang and Xin Qu* \\ Department of Neurosurgery, Xuanwu Hospital of Capital Medical University, Beijing, China
}

Objective: To determine the effect of the serum albumin level on admission in patients with spontaneous subarachnoid hemorrhage (SAH).

Methods: A total of 229 patients with SAH were divided into control and hypoalbuminemia groups. The serum albumin levels were measured. The data, including age, gender, co-existing medical conditions, risk factors, Hunt-Hess $(H-H)$ grade on admission, Glasgow coma score (GCS) on admission, complications during hospitalizations, length of hospital stay, length of intensive care unit (ICU) stay, in-hospital mortality, survival rate, outcome at discharge, and the 6-month follow-up outcome, were compared between the two groups.

Results: Older age, an increased number of patients who consumed an excess of alcohol, and a lower GCS on admission were findings in the hypoalbuminemia group compared to the control group $(p<0.001)$. The ratio of patients with $\mathrm{H}-\mathrm{H}$ grade I on admission in the hypoalbuminemia group was decreased compared to the control group $(p<0.05)$. Patients with hypoalbuminemia were more likely to be intubated, and have pneumonia and cerebral vasospasm than patients with a normal albumin level on admission $(p<0.001)$. Furthermore, the length of hospital and ICU stays were longer in the hypoalbuminemia group than the control group $(p<0.001)$. Hypoalbuminemia on admission significantly increased poor outcomes at discharge $(p<0.001)$. The number of patients with severe disability was increased and the recovery rate was decreased with respect to in-hospital outcomes in the hypoalbuminemia group than the control group $(p<0.001)$.

Conclusion: Hypoalbuminemia was shown to be associated with a poor prognosis in patients with SAH.

Keywords: albumin, spontaneous subarachnoid hemorrhage, Glasgow outcome scale, at admission, predictor

\section{INTRODUCTION}

Subarachnoid hemorrhage (SAH) is a severe cerebrovascular disease caused by blood flowing into the subarachnoid space after intracranial vascular rupture. Subarachnoid hemorrhage is clinically divided into traumatic and non-traumatic types. Non-traumatic SAH, also known as spontaneous $\mathrm{SAH}$, is a common and highly fatal stroke (1). The incidence of spontaneous SAH in China is 
approximately $2 / 100,000$ per year, accounting for $5-10 \%$ of all strokes $(2,3)$. Intracranial aneurysms are the main cause of spontaneous $\mathrm{SAH}$, accounting for approximately $85 \%$ of all cases. The clinical prognosis of SAH is relatively poor, and the mortality within 3 months of onset and the disability rate of surviving patients is also relatively high. At present, SAH is an acute hemorrhagic cerebrovascular disease that endangers human health $(1,4)$.

A low serum albumin level is associated with increased morbidity and mortality in various diseases (5-8). Albumin, a major component of plasma protein, has an important role in maintaining normal oncotic pressure and microvascular permeability (9-11). Hypoalbuminemia usually occurs in patients with acute ischemic stroke $(12,13)$. Furthermore, patients with intracerebral hemorrhage $(\mathrm{ICH})$ and concurrent hypoalbuminemia are also more susceptible to poor functional outcomes (14).

There are limited data and studies that have focused on the effect of hypoalbuminemia on the outcomes of patients with SAH. Specifically, long term follow-up studies with a large number of patients have been insufficient (15-20). Therefore, we determined the influence of a low serum albumin level at the time of admission in patients with spontaneous SAH.

\section{SUBJECTS AND METHODS}

\section{Subjects}

This retrospective study was approved by the Hospital Review Board. A total of 229 patients who were admitted to our Neurosurgery intensive care unit (ICU) with a diagnosis of spontaneous SAH from December 2017 to December 2018 were enrolled. The diagnosis of SAH met the diagnostic criteria of the 2015 edition of Chinese Guidelines for the Diagnosis and Treatment of Subarachnoid Hemorrhage (2). The inclusion criteria were as follows: (1) spontaneous onset; (2) the diagnostic criteria for SAH were met; (3) hospitalized within 7 days of onset; (4) serum albumin levels were closely monitored within $24 \mathrm{~h}$ after admission; (5) a head CT, lumbar puncture, and head and neck CTA/DSA/MRA examinations obtained after admission; and (6) complete medical records. The exclusion criteria were as follows: (1) died within $24 \mathrm{~h}$ after admission; (2) traumatic or spontaneous SAH undergoing craniotomy; (3) heart, lung, liver, kidney, and other vital organ dysfunction; (4) a history of severe coagulation dysfunction; (5) incomplete medical records; and (6) no serum albumin level obtained on admission.

\section{Determination of Serum Albumin Levels}

The serum albumin levels were measured at $24 \mathrm{~h}$ and 1 week after onset. The serum albumin level was commonly measured by recording a change in absorbance upon binding to a dye, such as bromocresol green or bromocresol purple (21, 22). Hypoalbuminemia was established when the serum albumin level was $<3.5 \mathrm{~g} / \mathrm{dl}$ (23). Patients were divided into control and hypoalbuminemia groups depending on the diagnosis of hypoalbuminemia on admission.

\section{Data Collection}

The baseline demographics (age and gender), other diseases (diabetes mellitus, hyperlipidemia, and coronary artery disease), risk factors [hypertension, smoking, and excessive alcohol consumption (24)], admission Hunt-Hess $(\mathrm{H}-\mathrm{H})$ grade (25), Glasgow coma score (GCS) on admission, complications during hospitalization [intubation, pneumonia, cerebral vasospasm, re-bleeding, and delayed cerebral ischemia (DCI)], length of hospital stay, length of ICU stay, in-hospital mortality, survival rate, outcomes at discharge, and 6-month follow-up outcomes were collected. Hypertension was diagnosed if the systolic blood pressure was $>140 \mathrm{mmHg}$ or the diastolic blood pressure was $>90 \mathrm{mmHg}$, or the patient was taking anti-hypertensive medications. Cerebral vasospasm was described as focal or diffuse temporarily narrowed vessel caliber due to the contraction of a smooth muscle in the wall of the arteries, which was detected by angiography, transcranial Doppler (TCD), magnetic resonance (MR), and CT (26). Rebleeding was identified as an acute clinical deterioration that was accompanied by evidence of rebleeding in the subarachnoid space, ventricular system, or brain parenchyma via a follow-up CT or an autopsy (27). Delayed cerebral ischemia (DCI) was defined as the development of new focal neurologic signs and/or a deterioration in the level of consciousness lasting for $>1 \mathrm{~h}$, or the appearance of new infarctions on CT or MRI (28-30).

\section{Outcome Measures}

Poor outcome was defined as in-hospital death, or transfer to hospice care or a nursing home facility [GOS scale $\leq 3$ (27)]. Serious complications included another or several potentially fatal diseases triggered during the course of the SAH. Furthermore, the 6-month follow-up outcomes were also recorded.

\section{Statistical Analysis}

Statistical analysis was performed by SPSS17.0 (International Business Machines, Corp., Armonk, NY, USA). The differences between the two groups were compared by a chi-square test or Fisher's exact test for categorical data and Student's $t$-test or Kruskal test for continuous data. Multivariable logistic or linear regression analysis was carried out to study the relationship between the albumin level and outcomes. All the multivariable regression models were repeated for the characteristic variables. A $p$-value $\leq 0.05$ was considered statistically different.

\section{RESULTS}

\section{General Clinical Characteristics}

Three hundred and fifteen patients with spontaneous SAH were admitted in the study. Among the 315 patients, 229 met the inclusion criteria, including 79 with normal albumin levels and 150 with hypoalbuminemia. The albumin level in the hypoalbuminemia group was decreased more than the control group $(p<0.001)$. The baseline data are shown in Table 1. The age in the hypoalbuminemia group was higher than the control group $(p<0.001)$. The number of patients that consumed excessive alcohol was greater and the mean GOS on admission was lower in the hypoalbuminemia group than 
TABLE 1 | Baseline characteristics of patients with spontaneous subarachnoid hemorrhage.

\begin{tabular}{|c|c|c|c|c|c|c|}
\hline Items & $\begin{array}{l}\text { All patients } \\
(n=229)\end{array}$ & $\begin{array}{l}\text { Low serum } \\
\text { albumin } \\
(n=150)\end{array}$ & $\begin{array}{l}\text { Normal serum } \\
\text { albumin } \\
(n=79)\end{array}$ & Test method & Test statistic & $p$-Values \\
\hline Age (years) & $55.53 \pm 13.11$ & $58.84 \pm 11.06$ & $49.24 \pm 14.39$ & Kruskal & 22.68 & $0.001^{\star}$ \\
\hline Gender (Males) & 103 (45.0\%) & $61(40.7 \%)$ & 42 (53.2\%) & Chi square & 3.27 & 0.071 \\
\hline Admission $\mathrm{H}-\mathrm{H}$ grade & & & & Chi square & 15.50 & $0.001^{\star}$ \\
\hline I & $59(25.8 \%)$ & 29 (19.3\%) & 30 (38\%) & Chi square & 3.52 & $0.002^{\star}$ \\
\hline$\|$ & 96 (41.9\%) & 57 (38.0\%) & 39 (49.4\%) & Chi square & 2.75 & 0.097 \\
\hline III & 33 (14.4\%) & 29 (19.3\%) & 4 (5.1\%) & Chi square & 8.54 & $0.003^{\star}$ \\
\hline IV & 26 (11.4\%) & 20 (13.3\%) & $6(7.6 \%)$ & Chi square & 1.69 & 0.193 \\
\hline V & 5 (2.2\%) & $5(3.3 \%)$ & $0(0 \%)$ & Chi square & 2.69 & 0.244 \\
\hline Hypertension & 129 (56.3\%) & 82 (54.7\%) & 47 (59.5\%) & Chi square & 0.49 & 0.484 \\
\hline Smoking & $90(39.3 \%)$ & 64 (42.7\%) & 26 (32.9\%) & Chi square & 2.06 & 0.151 \\
\hline Alcohol consumption & $49(21.4 \%)$ & 47 (31.3\%) & $2(2.5 \%)$ & Chi square & 25.52 & $0.001^{\star}$ \\
\hline Diabetes mellitus & 18 (7.9\%) & $11(7.3 \%)$ & 7 (8.9\%) & Chi square & 0.17 & 0.683 \\
\hline Hyperlipidemia & $6(2.6 \%)$ & $2(1.3 \%)$ & $4(5.1 \%)$ & Chi square & 2.82 & 0.213 \\
\hline Coronary artery disease & $15(6.6 \%)$ & $13(8.7 \%)$ & $2(2.5 \%)$ & Chi square & 3.18 & 0.074 \\
\hline Admission Glasgow coma score & $13.34 \pm 3.31$ & $12.86 \pm 3.65$ & $14.25 \pm 2.28$ & Kruskal & 15.45 & $<0.001^{*}$ \\
\hline
\end{tabular}

Measurement data are expressed as means \pm standard deviation (SD) and counting data are expressed as a percentage.

${ }^{*} p \leq 0.05$ : low serum albumin group vs. normal serum albumin group. $\mathrm{H}-\mathrm{H}$ grade, Hunt-Hess grade.

the normal albumin group $(p<0.001)$. Moreover, the ratio of patients with $\mathrm{H}-\mathrm{H}$ grade I on admission in the hypoalbuminemia group was lower than the control group (19.3 vs. $38 \%, p<$ $0.05)$. Furthermore, the ratio of patients with $\mathrm{H}-\mathrm{H}$ grade III on admission was higher in the hypoalbuminemia group than the control group (19.3 vs. $5.1 \%, p<0.05)$.

\section{Comparison of Prognosis Between the Two Groups}

Patients with hypoalbuminemia were more likely to be intubated, and have pneumonia and cerebral vasospasm than patients with a normal albumin level on admission ( 43.6 vs. $8.9 \%, p<0.001 ; 46.3$ vs. $13.9 \%, p<0.001$; and 50.7 vs. $32.9 \%, p=0.010$, respectively; Table 2). In addition, the length of hospital and ICU stays were longer in the hypoalbuminemia group than the control group (13.1 vs. 4.8 days, $p<0.001$; and 12.2 vs. 4.6 days, $p<0.001$, respectively). There was a significant increase in poor outcomes at the time of hospital discharge in the hypoalbuminemia group than the control group ( 30.0 vs. $2.5 \%, p<0.001$; OR $=33.4$; 95\% CI: 8.4-132.8). Considering the outcomes at the time of hospital discharge, the severe disability and recovery rates were significantly different between the two groups $(p<0.001)$, and this disparity persisted to the 6-month follow-up outcomes $(p=0.045 ; p=0.032)$. The leading causes of death during hospitalization and at the 6-month follow-up included severe primary diseases (mainly with severe and high grade SAH) and severe complications (rebleeding, severe cerebral vasospasm, hydrocephalus, epilepsy, and cerebral hernia). Fourteen patients were lost to follow-up by the 6-month evaluation. The number of patients decreased from 229 at the beginning of the study to 215 after follow-up.

\section{Independent Predictors}

After adjusting for age, gender, $\mathrm{H}-\mathrm{H}$ grade on admission, excessive alcohol consumption, coronary artery disease, and GCS on admission, we showed that hypoalbuminemia $(\mathrm{OR}=4.7, p=$ $0.006)$, pneumonia $(\mathrm{OR}=2.6, p=0.024)$, poor outcome $(\mathrm{OR}=$ $18.51, p=0.006$ ), length of hospital stay (effect size: 5.33 days, $p$ $<0.001$ ), and length of ICU stay (effect size: 4.29 days, $p=0.001$ ) were independent predictors for intubation (Table 3 ).

\section{DISCUSSION}

A low serum albumin level induces numerous health issues (5-8). The predictors of mortality and morbidity in patients with spontaneous SHA include age, hypertension, low GCS on admission, cerebral vasospasm, rebleeding, and DCI (24-29). In our study, most of these predictors, except for hypertension and DCI, were very different between the two groups, indicating that a multiple logistic regression analysis may be needed to further assess the correlation between hypoalbuminemia and outcomes at discharge.

The serum albumin level is a function of rates of synthesis, degradation, and distribution in the extracellular and intracellular spaces (9). Hypoalbuminemia may occur not only in patients with liver or kidney dysfunction and malnutrition, but also in the distribution or catabolism and the presence of inflammatory cytokines (31).

Only two-thirds of men 65-74 years of age with serum albumin levels $\geq 4.4 \mathrm{~g} / \mathrm{dl}$ have developed strokes compared to patients with albumin levels $\leq 4.2 \mathrm{~g} / \mathrm{dl}$ (32). The prevalence of renal dysfunction has been reported in $20-35 \%$ of patients with ischemic strokes and 30-46\% of patients with ICH (33). Therefore, hypoalbuminemia may in part be a result of complex cerebrorenal interactions and renal dysfunction (34-36). A low 
TABLE 2 | Correlation of serum albumin levels in patients with spontaneous subarachnoid hemorrhage.

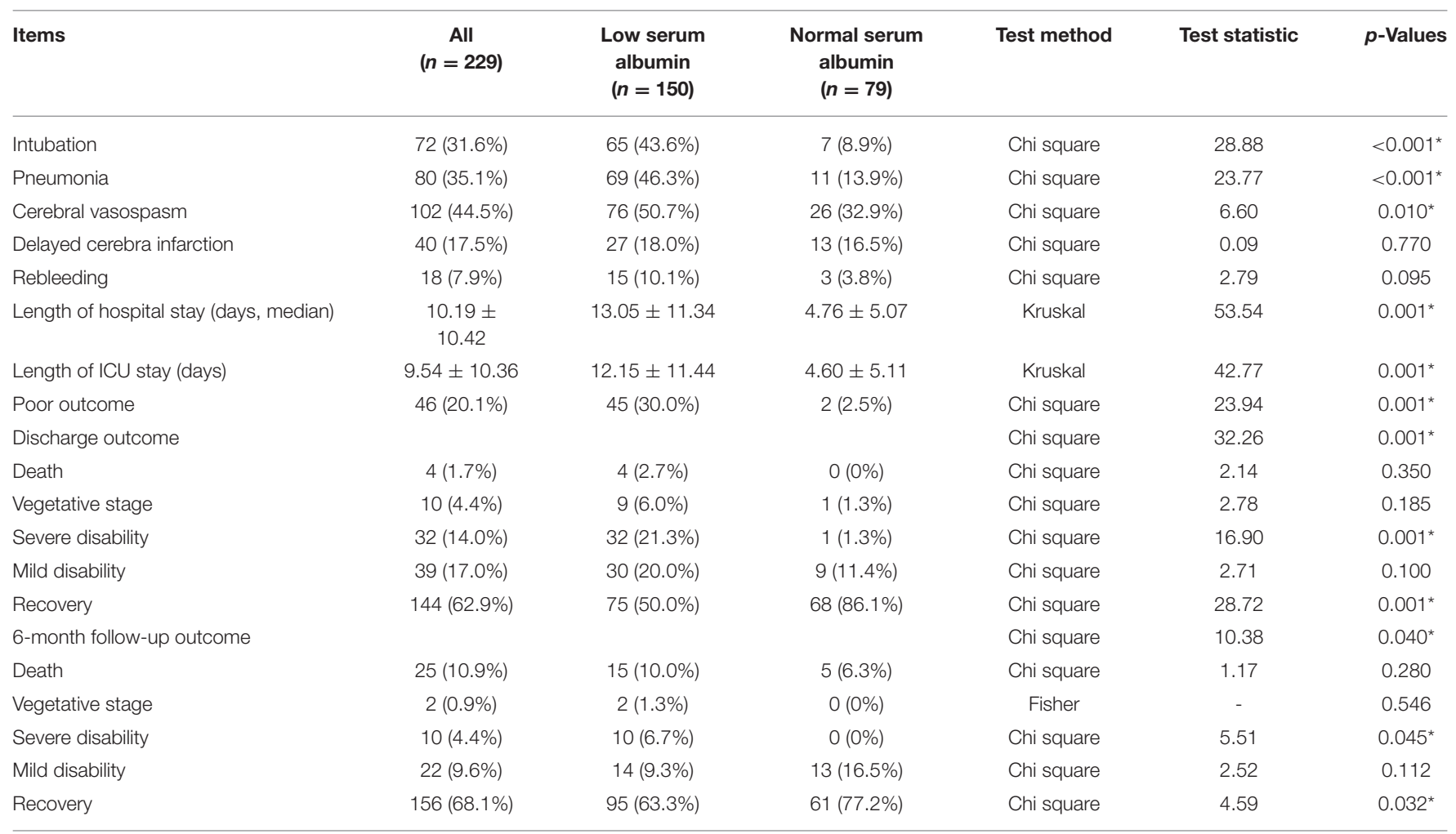

Measurement data are expressed as means \pm standard deviation (SD) and counting data are expressed as a percentage.

${ }^{*} p \leq 0.05$ : Iow serum albumin group vs. normal serum albumin group. ICU, intensive care unit.

albumin level could be a modifiable factor for patients with spontaneous SAH or other conditions.

There should be no major issue with the use of albumin in most developed countries and more advanced developing countries; however, for most low- and middle-income countries (LMICs), it is more difficult to assess serum chemistries, such as albumin, when it is even difficult to meet the requirements of essential medications and medical equipment (Is this change ok?). These issues are really difficult to overcome, and can only be handled according to the actual situation of the respective hospital departments.

There were some limitations in our study. The main limitation was the retrospective nature of the study. The simple laboratory test is needed to assess this alteration in the albumin level. Moreover, this was a single center study with a small sample size. The retrospective nature of data collection, the lack of information on the premorbid nutritional and volume status, the absence of serial albumin measurements, and long-term functional outcomes are all limitations. Larger prospective studies with serial measurements of serum albumin levels are needed. Therefore, the role of albumin supplementation in patients with spontaneous SAH are warranted.

\section{CONCLUSION}

In conclusion, we demonstrated that hypoalbuminemia on admission is a risk factor for poor outcome in patients with
TABLE 3 | Multivariable analysis of serum albumin levels in patients with spontaneous subarachnoid hemorrhage.

\begin{tabular}{lcc}
\hline Items & \multicolumn{2}{c}{ Low serum albumin } \\
\cline { 2 - 3 } & OR (95\% Cl) & $\boldsymbol{p}$-Values \\
\hline Intubation & $4.7(1.67-16)$ & $0.006^{*}$ \\
Pneumonia & $2.6(1.16-6.2)$ & $0.024^{*}$ \\
Vasospasm & $1.75(0.9-3.45)$ & 0.100 \\
Cerebral infarction & $0.97(0.4-2.41)$ & 0.954 \\
Rebreeding & $3.77(0.82-28.05)$ & 0.124 \\
Poor outcome & $18.51(3.41-349.03)$ & $0.006^{*}$ \\
& $\boldsymbol{\beta}(\mathbf{9 5} \% \mathbf{C l})$ & $\boldsymbol{p}$-Values \\
Length of hospital stay & $5.33(2.81-7.85)$ & $0.001^{*}$ \\
Length of ICU stay & $4.29(1.83-6.74)$ & $0.001^{*}$
\end{tabular}

${ }^{*} p \leq 0.05$.

spontaneous SAH. It is substantially relevant for prediction and prognosis of spontaneous SAH. Because measuring the serum albumin level is an affordable task, it may serve as an additional prediction marker in these patients. Larger prospective studies with serial measurements of serum albumin are required to identify the poor outcomes and thereby implement therapeutic interventions. 


\section{DATA AVAILABILITY STATEMENT}

The raw data supporting the conclusions of this article will be made available by the authors, without undue reservation.

\section{ETHICS STATEMENT}

The studies involving human participants were reviewed and approved by Xuanwu Hospital, Capital Medical University Committee. The patients/participants provided their written informed consent to participate in this study. Written informed

\section{REFERENCES}

1. Qiang D, Ming L, Chuanqiang P, Haibo C, Shengdi C, Liying C. Chinese guideline for diagnosis and treatment of subarachnoid hemorrhage 2015. Chin J Neurol. (2016) 3:182-91.

2. He Cheng. Clinical Observation on Treatment of Traumatic Subarachnoid Hemorrhage With Acupuncture And Medicine. Guangzhou University of traditional Chinese Medicine (2015).

3. Neifert SN, Chapman EK, Martini ML, Shuman WH, Schupper AJ, Oermann EK, et al. Aneurysmal subarachnoid hemorrhage: the last decade. Transl Stroke Res. (2020) 12: 428-46. doi: 10.1007/s12975-020-00867-0

4. Fiebach JB, Schellngfr PD, Geletneky K, et al. MRI in acute subarachnoid hemorrhage; findings with a standardised stroke protocol. Neuroradiology. (2004) 1:44-8. doi: 10.1007/s00234-003-1132-8

5. McCluskey A, Thomas AN, Bowles BJ, Kishen R. The prognostic value of serial measurements of serum albumin concentration in patients admitted to an intensive care unit. Anaesthesia. (1996) 51:724-7. doi: 10.1111/j.1365-2044.1996.tb07883.x

6. Ryan TA, Rady MY, Bashour CA, Leventhal M, Lytle B, Starr NJ. Predictors of outcome in cardiac surgical patients with prolonged intensive care stay. Chest. (1997) 112:1035-42. doi: 10.1378/chest.112.4.1035

7. Becker BN, Becker YT, Heisey DM, Leverson GE, Collins BH, Odorico JS, et al. The impact of hypoalbuminemia in kidney-pancreas transplant recipients. Transplantation. (1999) 68:72-5. doi: 10.1097/00007890-19990715000014

8. Vincent JL, Dubois MJ, Navickis RJ, Wilkes MM. Hypoalbuminemia in acute illness: is there a rationale for intervention? A meta-analysis of cohort studies and controlled trials. Ann Surg. (2003) 237:31934. doi: 10.1097/01.SLA.0000055547.93484.87

9. Nicholson JP, Wolmarans MR, Park GR. The role of albumin in critical illness. Br J Anaesth. (2000) 85:599-610. doi: 10.1093/bja/85.4.599

10. Demling RH. Effect of plasma and interstitial protein content on tissue edema formation. Curr Stud Hematol Blood Transfus. (1986) 53:3652. doi: $10.1159 / 000413163$

11. Jorgensen KA, Stoffersen E. On the inhibitory effect of albumin on platelet aggregation. Thromb Res. (1980) 17:13-8. doi: 10.1016/0049-3848(80) 90289-3

12. Dziedzic T, Pera J, Slowik A, Gryz-Kurek EA, Szczudlik A. Hypoalbuminemia in acute ischemic stroke patients: frequency and correlates. Eur J Clin Nutr. (2007) 61:1318-22. doi: 10.1038/sj.ejcn.1602643

13. Cho YM, Choi IS, Bian RX, Kim JH, Han JY, Lee SG. Serum albumin on admission for prediction of functional outcome in ischaemic stroke patients. Neurol Sci. (2008) 29:445-9. doi: 10.1007/s10072-008-1024-0

14. Limaye K, Yang JD, Hinduja A. Role of admission serum albumin levels in patients with intracerebral hemorrhage. Acta Neurol Belg. (2016) 116:2730. doi: 10.1007/s13760-015-0504-2

15. Kapoor A, Dhandapani S, Gaudihalli S, Dhandapani M, Singh H. Mukherjee KK. Serum albumin level in spontaneous subarachnoid haemorrhage: more than a mere nutritional marker! Br J Neurosurg. (2018) 32:4752. doi: 10.1080/02688697.2017.1344615

16. Behrouz R, Godoy DA, Topel CH, Birnbaum LA, Caron JL, Grandhi R, et al. Early hypoalbuminemia is an independent predictor of mortality consent was obtained from the individual(s) for the publication of any potentially identifiable images or data included in this article.

\section{AUTHOR CONTRIBUTIONS}

FS and HZ: designed the project. FS, HZ, WC, MQ, and NW: were responsible for experiments data collection and analysis, and manuscript writing. XQ revised the manuscript. All the authors participated in the review of the manuscript. in aneurysmal subarachnoid hemorrhage. Neurocrit Care. (2016) 25:2306. doi: 10.1007/s12028-016-0259-5

17. Xie Y, Liu W, Zhang X, Wang L, Xu L, Xiong Y, et al. Human albumin improves long-term behavioral sequelae after subarachnoid hemorrhage through neurovascular remodeling. Crit Care Med. (2015) 43:e4409. doi: $10.1097 / \mathrm{CCM} .0000000000001193$

18. Wang L, Li M, Xie Y, Xu L, Ye R, Liu X. Preclinical efficacy of human albumin in subarachnoid hemorrhage. Neuroscience. (2017) 344:25564. doi: 10.1016/j.neuroscience.2016.12.033

19. Suarez JI, Shannon L, Zaidat OO, Suri MF, Singh G, Lynch G, et al. Effect of human albumin administration on clinical outcome and hospital cost in patients with subarachnoid hemorrhage. J Neurosurg. (2004) 100:58590. doi: 10.3171/jns.2004.100.4.0585

20. Suarez JI, Martin RH, Calvillo E, Dillon C, Bershad EM, Macdonald RL, et al. The Albumin in Subarachnoid Hemorrhage (ALISAH) multicenter pilot clinical trial: safety and neurologic outcomes. Stroke. (2012) 43:68390. doi: 10.1161/STROKEAHA.111.633958

21. Doumas BT, Watson WA, Biggs HG. Albumin standards and the measurement of serum albumin with bromcresol green. Clin Chim Acta. (1971) 31:87-96. doi: 10.1016/0009-8981(71)90365-2

22. Dumas BT, Watson WA, Biggs HG. Albumin standards and the measurement of serum albumin with bromcresol green. Clin Chim Acta. (1997). 258:2130. doi: 10.1016/S0009-8981(96)06447-9

23. Gatta A, Verardo A, Bolognesi M. Hypoalbuminemia. Intern Emerg Med. (2012) 7(Suppl 3):S193-199. doi: 10.1007/s11739-012-0802-0

24. Macdonald RL, Schweizer TA. Spontaneous subarachnoid haemorrhage. Lancet. (2017) 389:655-66. doi: 10.1016/S0140-6736(16)30668-7

25. Hunt WE, Hess RM. Surgical risk as related to time of intervention in the repair of intracranial aneurysms. J Neurosurg. (1968) 28:1420. doi: 10.3171/jns.1968.28.1.0014

26. Baggott CD, Aagaard-Kienitz B. Cerebral vasospasm. Neurosurg Clin N Am. (2014) 25:497-528. doi: 10.1016/j.nec.2014.04.008

27. Broderick JP, Brott TG, Duldner JE, Tomsick T, Leach A. Initial and recurrent bleeding are the major causes of death following subarachnoid hemorrhage. Stroke. (1994) 25:1342-7. doi: 10.1161/01.STR.25.7.1342

28. Vergouwen MDI, Participants in the international multi-disciplinary consensus conference on the critical care management of subarachnoid hemorrhage. Vasospasm versus delayed cerebral ischemia as an outcome event in clinical trials and observational studies. Neurocrit Care. (2011) 15:30811. doi: 10.1007/s12028-011-9586-8

29. D'Souza S. Aneurysmal subarachnoid hemorrhage. J Neurosurg Anesthesiol. (2015) 27:222-40. doi: 10.1097/ANA.0000000000000130

30. Fragata I, Canhao P. Imaging predictors of outcome in acute spontaneous subarachnoid hemorrhage: a review of the literature. Acta Radiol. (2019) 60:247-59. doi: 10.1177/0284185118778877

31. Gopal DM, Kalogeropoulos AP, Georgiopoulou VV, Tang WW, Methvin A, Smith AL, et al. Serum albumin concentration and heart failure risk. Am Heart J. (2010) 160:279-85. doi: 10.1016/j.ahj.2010.05.022

32. Gillum RF, Ingram DD, Makuc DM. Relation between serum albumin concentration and stroke incidence and death: the NHANES I Epidemiologic Follow-up Study. Am J Epidemiol. (1994) 140:876-88. doi: 10.1093/oxfordjournals.aje.a117176 
33. Toyoda K, Ninomiya T. Stroke and cerebrovascular diseases in patients with chronic kidney disease. Lancet Neurol. (2014) 13:823-33. doi: 10.1016/S1474-4422(14)70026-2

34. Toyoda K. Cerebrorenal interaction and stroke. Contrib Nephrol. (2013) 179:1-6. doi: 10.1159/000346944

35. Yahalom G, Schwartz R, Schwammenthal Y, Merzeliak O, Toashi M, Orion $\mathrm{D}$, et al. Chronic kidney disease and clinical outcome in patients with acute stroke. Stroke. (2009) 40:1296-303. doi: 10.1161/STROKEAHA.108.520882

36. Jennett B, Bond M. Assessment of outcome after severe brain damage. Lancet. (1975) 1:480-4. doi: 10.1016/S0140-6736(75)92830-5

Conflict of Interest: The authors declare that the research was conducted in the absence of any commercial or financial relationships that could be construed as a potential conflict of interest.
Publisher's Note: All claims expressed in this article are solely those of the authors and do not necessarily represent those of their affiliated organizations, or those of the publisher, the editors and the reviewers. Any product that may be evaluated in this article, or claim that may be made by its manufacturer, is not guaranteed or endorsed by the publisher.

Copyright (c) 2021 Shang, Zhao, Cheng, Qi, Wang and Qu. This is an open-access article distributed under the terms of the Creative Commons Attribution License (CC $B Y)$. The use, distribution or reproduction in other forums is permitted, provided the original author(s) and the copyright owner(s) are credited and that the original publication in this journal is cited, in accordance with accepted academic practice. No use, distribution or reproduction is permitted which does not comply with these terms. 